\title{
Automation of technological process of obtaining pectin
}

\author{
Anna I. Belousova ${ }^{1, *}$, and Lyudmila V. Donchenko ${ }^{1}$ \\ ${ }^{1}$ FSBEI HE Kuban State Agrarian University named after I.T.Trubilin, Krasnodar, Russian Federation
}

\begin{abstract}
The article discusses the feasibility of automation of the process of obtaining liquid pectin for the production of functional beverages to ensure the desired quality and physiological properties. There were presented comparative differences between traditional operator panels and workstations. There was substantiated the expediency of control and regulation of main technological parameters of the process of extraction of pectin substances. There was described the algorithm of evaluating the results of real-time monitoring of the process.
\end{abstract}

Practically in many countries of the world the increasing interest is paid to production of so-called healthy food. In particular, there is a growing interest in drinks that can not only quench your thirst, but also have a positive impact on various systems of a human body and its condition as a whole. Functional energetic drinks of general health-improving action are designed to stimulate mental activity, relaxation, prevention of cholesterol metabolism and others. The concept of "Drinks for health" has become fundamental for many European manufacturers and an effective brand that allows you to compete successfully in the market. According to the market research, consumers prefer functional drinks made from natural, environmentally friendly raw materials and ingredients. In this regard, the production of fruit and vegetable juices and drinks with the addition of pectin substances is promising.

The value of such products is determined by the maximum preservation of biologically active components of raw materials. The advantages of plant products are that they are rich sources of vitamins, antioxidants, minerals and phytoelements. A promising direction is also the expansion of the range of pectin-containing beverages with desired physiological properties.

A successful technological solution is the production of such beverages by combining a given ratio with a controlled chemical composition of fruit and vegetable drinks and liquid pectin $[1,2]$.

The use of vegetable raw materials rich in vitamins, trace elements, dietary fibers, biologically active substances, enhances the nutritional and biological value of such beverages, and the presence in the product of pectin substances introduced in the form of pectin concentrate, allows us to refer the product to the functional group. The carbohydrate complex of vegetable cultures is characterized by a high content of non-digestible

\footnotetext{
${ }^{*}$ Corresponding author:
} 
polysaccharides, inulin accumulates in the composition of individual vegetables, there are soluble fruit oligosaccharides, $\beta$ carotene, pantothenic acid and other prebiotics, which suggests that they have bifidogenic properties [3].

The purpose of our work is to study the features and stability of the process of producing liquid pectin with the prospect of its use in the production of probiotic beverages.

It is known that currently the requirements for the quality of food products, including beverages, determine not only the type of equipment that performs a particular operation, but also the conditions, principles and methods of its work, as well as the presence of different types and brands of machines and devices used in industry. Automation systems (SA) and automatic process control systems (APCS) applied in the food industry are different and diverse in types, means of communication (communication parameters), interfaces, type of signal processing, etc.

At the same time, until recently, operator consoles were widely used as a means of monitoring and controlling the technological process (TP). As a rule, they are installed directly in the shop where the TP is held, which often leads to the large size of the operator panels, a lot of individual modules needed to control the system, and at the same time they are little functional compared to workstations, which are increasingly used [4, 5].

In our opinion, the widespread use of industrial computers and industrial workstations in food enterprises can significantly facilitate the solution of a number of automation tasks. So, in comparison with traditional operator panels, workstations will allow:

- $\quad$ transfer data automatically to and from the operator;

- $\quad$ keep and analyze information;

- $\quad$ give information in appropriate format;

- $\quad$ manage with a processor by command information of an operator;

- $\quad$ manage by the process independently;

- $\quad$ cost decrease;

- labor productivity increase.

The obvious advantage of workstations is also in the replacement of control panels with complex wiring, providing a significant increase in flexibility. The progress of the process is displayed graphically, allowing you to assess the status of the system at a glance. To ensure the safety of food production, workstations are able to send an alarm signal repeatedly. Alarms can be quickly given by means of graphics and automatic messages to the operator. The workstation can be a useful tool for solving the problems of processing information about the TP, reducing to a minimum the downtime of the food production line at the enterprises with deviations of the quality parameters of raw materials, semi-finished products and finished products. Information from different parts of the process can be presented in real time, stored or made available for management via the network. The workstation allows you to organize interaction with any equipment, from software and logic controllers to universal computers. It is obvious that the workstation must have a computational module of the computer for processing TP [6].

Depending on the customer, it may consist of a single computer or a network of computers.

It should be noted that industrial computers are very different from office computers. Their work should be focused on tough operating conditions (presence of dust, extreme mechanical and climatic influences, as well as intense electromagnetic radiation). In addition, they must be equipped with high-speed cooling fans and air filters. This will ensure a constant flow of air to create excess air pressure inside the frame, which ensures reliable operation of IPC even at high ambient temperatures. Most industrial computers are also provided with locking devices for circuits and protected from bumps of compartments for disk drives. Most industrial computers are made of high-quality steel with a special coating to protect against electromagnetic radiation. The power sources are insensible to 
voltage emissions in the net, over-voltage and voltage fluctuations providing stable output voltage.

In the context of increased competition, the ability to access information about the production process from any level of the organizational structure of an enterprise is one of the most important requirements for automated control systems, which is especially important for pectin production.

Standard operating systems allow you to choose software development tools and application software from a variety of independent vendors. The open architecture allows users running these operating systems to access servers such as Windows NT that use database management systems (DBMS) such as Microsoft SQL Server and other popular DBMS.

Development of the program can be done on a regular computer. It is object-oriented and can easily model and track each stage of technological operations of pectin production, allow to regulate, control and improve its production process, save money by reducing the consumption of time and resources. The majority of this type of programs comes with many different means of interaction with enterprise software systems of data processing, including mechanisms for dynamic data exchange (DDE), linking and embedding of objects (OLE Automation) and exchange on a local area network using protocols such as IPX. Functions of open databases interaction (ODBI) are built in between databases to implement the relations.

The purpose of automation of the stability process of pectin production is improvement of the quality of finished products on the basis of forecasting the quantitative content of pectin substances in the extract including their fractionalization in raw materials and violations of technological parameters in the process of obtaining. The selection and systematization of requirements to the development of automated intelligent expert systems of AIIS control of the stability of the pectin production process can be considered on the example of technological parameters control in the process of pectin obtaining.

The selection and systematization of requirements were carried out in the process of analyzing the goals and objectives of control automation on the basis of a multidisciplinary approach.

The structure of the automated temperature control system of the extraction process, one of the main technological parameters, as an object of automation includes extraction devices (control object), temperature measuring devices, recording devices as part of measuring devices with registration of information in electronic form, computer implementation of the control system. The construction of a network of temperature sensors is made in accordance with the control technological points and unacceptable deviations and is determined by the length and branching of a thermal circuit. The main requirements for the development of the automated temperature control structure are as follows:

- $\quad$ availability/construction of the scheme of extraction devices;

- calculation of sensors in accordance with specifications showed to the process of hydrolysis-extraction of pectin substances for the purpose of objectivity of the results in the measurement of temperature;

- $\quad$ computerization of the core of an automated intelligent system;

- availability of information-analytical subsystems and output of the result and formation of the control signal stimulus;

- $\quad$ openness to hardware upgrades.

The intellectual component of ACS is a reliable probability of reducing the quantitative content of pectin substances in violation of the temperature regime of extraction and the possibility of a significant deterioration in the quality of liquid pectin. The main parameters under study are the quantitative content (mass fraction) of pectin substances in the extract 
and their main analytical characteristics (degree of esterification, content of free carboxyl groups, methoxyl and acetyl components), temperature and time of the extraction process.

Scientific and objectivity in the creation of intellectual components of AIS is achieved by meeting the requirements:

- formation of a database of quantitative content of pectin substances in the extract based on the analysis of experimental results of the study of their fractional composition in processed raw materials and references;

- development of a predictive mathematical model of the growth of the mass fraction of pectin in the extract.

The operation of the AIES provides information on the results of real-time monitoring of the process temperature, including episodes of unplanned temperature variability, including changed temperature parameters and the duration of emergency.

Therefore, the functionality of the system should provide:

- reading the temperature parameters of the extractant, water and steam from the sensors in the context of the time and place of the process;

- $\quad$ graphic picture of temperature mode of pectin extraction process;

- $\quad$ processing of received digital and visual information, mathematical calculations;

- $\quad$ issuance of control or information signals;

- $\quad$ image of kinetic curve of growth of the quantitative content of pectin substances;

- $\quad$ analysis of compliance of measurements in TCP (technological control points) with established critical limits;

- $\quad$ emergency notification of responsible persons;

- $\quad$ documentation of procedures;

- $\quad$ preparation and analysis of reporting forms, sending reports;

- $\quad$ archiving and storage of information.

Monitoring of temperature regimes involves measuring the temperature of the process in the extraction apparatus during the pectin extraction process with a portable device installed in place, with remote transmission of readings.

Requirements to the functionality of portable temperature measuring devices:

- $\quad$ large memory capacity, high accuracy;

- $\quad$ availability of autonomous power supply, ability to replace the battery;

- implementation of simultaneous (parallel) temperature measurement inside the extraction apparatus and jacket by external and internal sensors (probes) of the apparatus;

- record data at remote unattended installations (remote information retrieval), in particular, with sensors located in the technological apparatus;

- $\quad$ compliance with the operating mode of measurement when reading data, thereby ensuring the continuity of control;

- the ability to connect to a computer and transfer monitoring data to the database on the computer of the operator and (or) the server, allowing using the appropriate computer program to create reports, tables and graphs based on them.

In accordance with the current legislation, the technical means of ACS used in the interaction of ACS with other systems should be compatible in interfaces with corresponding technical means of these systems and used communication systems [7].

Software implementation of control system automation is possible if the following requirements are met:

- $\quad$ free and/or open source software;

- modifiability;

- $\quad$ client-server model;

- $\quad$ modularity of construction and ease of use;

- web access; 
- $\quad$ session data processing.

Evaluation of monitoring results should be carried out in real time according to the algorithm:

- Visualization of the temperature curve graph and the kinetic growth curve of the quantitative content of pectin in the extract on the monitor screen.

- In case of temperature deviations exceeding the permissible, or reaching critical values of its increase or decrease: the nature and color of the temperature curve and (or) the curve of the quantitative content of pectin in the extractant, an audio signal is activated; a notification message is sent to the operator; an appropriate entry is made in the emergency $\log$.

- Ability of making decision and issuing a control signal to: a continuation of the process of extraction of pectin substances in the prescribed temperature regime; the optimization of the parameters of temperature; the termination of the process.

- Ability to create automatic reports on accident-free operation or frequency, nature and consequences of emergencies, archives.

- Forms of the created documents must comply with the requirements of the standards of the unified documentation system (UDS) or regulatory and technical documents of the customer's office.

Conclusions: formation of adequate requirements for AIS based on the requirements of regulations, multidisciplinary approach, data of special and scientific literature and research results increases the degree of:

- $\quad$ rationality of the structure of the AIS, the use of technical means and measuring instruments;

- $\quad$ objectivity of control results and their documentation;

- adequacy (objectivity, correctness, completeness, timeliness) of management decisions.

\section{References}

1. Sobol I.V. Peculiarities of analytical characteristics of pectin's extracted from sunflower hearts /I.V. Sobol, L.V. Donchenko, L.Y. Rodionova, A.G. Koshchaev, A.V.Stepovoy // Asian Journal of Pharmaceutics, 2017. T. 11, № 1. - S 97-100.

2. Ogneva O.A Pectin-containing beverages with probiotic properties / О.А. Огнева, L.V. Donchenko // Multidisciplinary network electronic scientific journal of Kuban State Agrarian University, 2015, № 107. - p. 333-341.

3. Degtyarev L.S. Properties and structure of galacturonic acid in pectin production technology. Proceedings of Higher educational institutions. / L.S. Degtyarev, M.P. Kupchik, L.V. Donchenko, O.V.Bogdanova // Food Technology, 2002, № 4. - p. 1517.

4. Ily'ina I.A. Features of technology of pectin obtaining with high prolonged properties / I.A. Ily'ina, L.V. Donchenko, Z.G.Zemskova // Messenger of Russian Agricultural Academy, 2003, № 3. - p. 8-10.

5. Azimova S.T. To wards food security through application of novel scientific findings / S.T. Azimova, M.Z. Kizatova, S.O. Akhmetova, L.V. Donchenko, A.M.Admayeva // Journal of Security and Sustainability Issues, 2017. T. 6, № 4. - p. 719-728.

6. Belousova, A. I. INNOVATION SOLUTIONS IN WORKING OUT OF SPECIALIZED NUTRITION BICOMPONENT BEVERAGE TECHNOLOGY [Text] / A.I. Belousova, L.V. Donchenko // in the book: food technologies: researches, 
innovations: materials 1 of national scientific-practical conference, Kerch, 2018: KSMTU, IMBI, AzNIIRH, 2018. p. 6-8.

7. Donchenko L.V.: main properties, production and application / L.V. Donchenko, G.G. Firsov. M.: DeLi print, 2007. - 276 p. 